\title{
Análise das relações taxonômicas e sistemáticas entre espécies de triatomíneos (Hemiptera, Reduviidae) de colônias mantidas pelo Serviço Especial de Saúde de Araraquara, inferida de sequiências do 16S rDNA mitocondrial
}

\author{
Walter Ceretti Junior ${ }^{1}$, Daniel Pagotto Vendrami ${ }^{1}$, João Molina Gil², José Maria Soares Barata ${ }^{1}$ \\ \& Mauro Toledo Marrelli ${ }^{1}$
}

${ }^{1}$ Departamento de Epidemiologia, Faculdade de Saúde Pública, Universidade de São Paulo, Av. Dr. Arnaldo 715, 01246-904 São Paulo-SP, Brasil. mmarelli@usp.br

${ }^{2}$ Serviço Especial de Saúde de Araraquara (SESA), Universidade de São Paulo, Rua Itália 1622, Centro, 14801-350 Araraquara, São Paulo-SP, Brasil.

\begin{abstract}
Taxonomic and systematic analysis among triatomine bug species (Hemiptera, Reduviidae) from colonies of the Special Health Service of Araraquara (SESA), inferred from 16S rDNA mitocondrial sequences. Nucleotide sequences of mitocondrial 16S rDNA gene were analyzed in 14 populations of triatomine bugs from colonies maintained by SESA, Araraquara, São Paulo, Brazil, comparing them with sequences of same gene found in the GenBank. The fragments varied from 311 to $317 \mathrm{bp}$, with low intra-specific genetic distance ( $0 \%$ to $0.6 \%$ ), with exception of the relationship among Triatoma sordida specimens (1\%) and T. brasiliensis specimens $(1.3 \%)$ that were attributed to different geographical populations. The paraphily of Rhodniini and Panstrongylus genus was evidenced by this analyses, confirming previous results between these and the narrow relationships of $R$. prolixus with $R$. robustus and of $T$. infestans with $T$. platensis. The relationship between $T$. maculata and $T$. pseudomaculata has not been resolved, since these taxa appeared both in monophyly and as in paraphyly: T. pseudomaculata (SESA) is grouped with T. maculata (sequence of GenBank) and the associated T. brasiliensis (SESA), while T. maculata (SESA) appears grouped with T. pseudomaculata of SESA and GenBank. The results evidence the utility of the gene $16 \mathrm{~S}$ as molecular marker in the species of triatomine bugs and its importance in systematic and taxonomy questions. There is a necessity of new studies including new molecular markers associated with classic systematic characters of morphology, ecology and behavior to adjust systematic decisions since those will be not of only systematic impact but, for new strategies of control.
\end{abstract}

KEYWORDS. Triatominae; Chagas disease vectors; systematic; 16S rDNA.

RESUMO. Análise das relações taxonômicas e sistemáticas entre espécies de triatomíneos (Hemiptera, Reduviidae) de colônias mantidas pelo Serviço Especial de Saúde de Araraquara (SESA), inferida de sequiências do 16S rDNA mitocondrial. Foram analisadas seqüências de nucleotídeos do gene 16S do rDNA mitocondrial em 14 populações de triatomíneos mantidos em colônias no insetário SESA de Araraquara- SP, comparando-as com sequiências do mesmo gene disponíveis no GenBank. Os fragmentos variaram de 311 a 317 pb com baixa variação intra-específica entre as distâncias genéticas ( $0 \%$ a $0,6 \%$ ), exceto para os relacionamentos entre espécimes de Triatoma sordida (1\%) e espécimes de T. brasiliensis $(1,3 \%)$ atribuídos a populações geográficas diferentes. A parafilia de Rhodniini e do gênero Panstrongylus foi evidenciada pelas analises, confirmando resultados anteriores entre estes e os estreitos relacionamentos de $R$. prolixus com $R$. robustus e de T. infestans e T. platensis. O relacionamento entre T. maculata e T. pseudomaculata não foi solucionado, uma vez que, esses táxons apareceram tanto em monofilia quanto em parafilia: T. pseudomaculata (SESA) está agrupado com T. maculata (seqüência do GenBank) e associados a $T$. brasiliensis (SESA), enquanto T. maculata (SESA) aparece agrupado com T. pseudomaculata do SESA e do GenBank. Os resultados evidenciam a utilidade do gene 16S como marcador de espécies de triatomíneos e sua importância em questões de sistemática e taxonomia. Há necessidade de novos estudos envolvendo outros marcadores associados a caracteres sistemáticos clássicos de morfologia, ecologia e comportamento para decisões sistemáticas adequadas uma vez, que teriam impacto não apenas sistemático mas, para as estratégias de controle.

PALAVRAS-CHAVE. Triatominae; Vetores da doença de Chagas; sistemática; 16S rDNA.

A subfamília Triatominae de Reduviidae comporta hoje, cerca de 142 espécies ocorrentes e uma fóssil, distribuídas em 18 gêneros. Esses insetos, hematófagos estritos em todas as fases da vida são reconhecidos vetores da doença de Chagas, parasitemia endemicamente distribuída entre as latitudes $40^{\circ} \mathrm{N}$ e $46^{\circ} \mathrm{S}$ do Sul dos EUA à Patagonia Argentina (Lent \& Wygodzinsky 1979; Schofield 1988; Galvão et al. 2003; Forero et al. 2004; Poinar 2005; Costa et al. 2006; Galvão \& Angulo
2006; Sandoval et al. 2007; Costa \& Felix 2007; Martinez et al. 2007).

Essa enfermidade é considerada um dos mais importantes problemas de Saúde na América Latina, com cerca de 9 a 14 milhões de indivíduos chagásicos, 60 milhões vivendo em risco e cerca de 20.000 caso/ano em 18 países da América do Sul e Central (W. H. O. 1990, 1991; Schmuñis, 1999; W. H. O. 2002; Schofield et al. 2006). 
Morfologicamente os triatomíneos em sua maioria são de fácil distinção, no entanto alguns táxons necessitam de ferramentas mais sutis para uma adequada distinção (Schofield 1988; Barata 1998; Carcavallo et al. 2001; Jurberg 2003). É o caso dos "complexos" de espécies crípticas, $R$. robustus e $T$. sordida, entre outras (Monteiro et al. 2001).

$\mathrm{O}$ uso de marcadores moleculares para fins de análise cladística em Triatominae, auxilia na resolução desses impasses sistemáticos e também acirrou as questões sobre as hipóteses de origem monofilética e parafilética do grupo (Jurberg 2003; Scheafer 2003; 2005).

Para inferências filogenéticas de populações e problemas relativos ao status específico de alguns táxons o gene $16 \mathrm{~S}$ do DNA mitocondrial tem sido bastante útil (Abad-Franch \& Monteiro 2005). No GenBank constatamos que já existe uma quantidade significativa de seqüências disponíveis deste gene para vários insetos, inclusive Triatominae.

O Serviço Especial de Saúde de Araraquara (SESA) pertencente à Faculdade de Saúde Pública da Universidade de São Paulo, Brasil, mantém um insetário constituído a partir da desativação do biotério existente no Departamento de Epidemiologia, na unidade matriz, na década de 1980. Conta atualmente com cerca 31 espécies de triatomíneos dos gêneros Triatoma, Rhodnius e Panstrongylus distribuídos em 140 colônias de diversas procedências nacionais e internacionais que são utilizadas por pesquisadores locais e de instituições de outros estados e nações. No entanto, somente umas poucas colônias foram pauta de estudos moleculares.

O presente trabalho tem por finalidade analisar sequiências do gene 16S do rDNA mitocondrial dos triatomíneos mantidos nas colônias do SESA e compará-las àquelas disponíveis no GenBank no intuito de fornecer novos dados moleculares que possibilitarão análises mais robustas futuramente.

\section{MATERIALE MÉTODOS}

Procedência dos Triatomíneos. Foram analisados 39 triatomíneos representantes de 14 espécies dos gêneros Panstrongylus, Rhodnius e Triatoma, adultos (machos e/ou fêmeas) e ninfas de V estádio, conforme Tabela 1. Esses indivíduos foram procedentes de colônias mantidas pelo insetário do Serviço Especial de Saúde de Araraquara (SESA), da Universidade de São Paulo.

De algumas espécies testamos mais de uma procedência (CTAs), caso de: $R$. prolixus Stål 1859 (3), P. megistus (Burmeister 1835) (5), T. infestans (Klug 1834) (8), T. pseudomaculata Correa \& Espinola 1964(3), T. rubrovaria Blanchard 1843 (2) e T. sordida Stål 1859 (2). Os trabalhos foram executados no Laboratório de Entomologia em Saúde Pública (LESP) da Faculdade de Saúde Pública da Universidade de São Paulo.

Os exemplares enviados semanalmente pelo insetário do SESA foram acondicionados em Tubos tipo Falcon de $50 \mathrm{ml}$, devidamente identificados e conservados em freezer a $-70^{\circ} \mathrm{C}$ até o momento da extração do DNA total.

Sequiências do gene $16 \mathrm{~S}$ de alguns exemplares de 18 outras espécies de triatomíneos dos gêneros Rhodnius, Psammolestes Bergroth, 1991, Triatoma, Panstrongylus, Eratyrus Stål, 1859, Dipetalogaster Usinger, 1939, Mepraia Mazza, Gajardom \& Jörg, 1940 e Linschosteus Distant, 1904 foram obtidas do GenBank, e estão listados na Tabela 2 com os respectivos números de acesso.

Extração do DNA total. Os exemplares obtidos foram mortos por congelamento a $-70^{\circ} \mathrm{C}$. O DNA genômico foi extraído individualmente utilizando o método de extração fenolclorofórmio, (Zahler \& Roth 1995) adaptado para triatomíneos por Lyman et al. (1999). De cada exemplar adulto retirou-se os três pares de pernas e a cabeça. As ninfas foram processadas integralmente.

O DNA foi obtido por meio da maceração individual, com pistilo, das peças anatômicas acima, em microtubos de $1,5 \mathrm{ml}$ nos quais se adicionou $150 \mathrm{ml}$ tampão de lise (Tris- $\mathrm{HCl} 10 \mathrm{mM}$, SDS 20\%, EDTA $50 \mathrm{mM}$ ) + 0,8 $\mathrm{ml}$ de proteinase $\mathrm{K} 20 \mathrm{ng} / \mathrm{ml}+$ $1,0 \mathrm{ml}$ de RNAse $10 \mathrm{ng} / \mathrm{ml}$. Desta forma obteve-se um homogenado que foi submetido a quatro ciclos de congelamento (em $\mathrm{N}_{2}$ líquido) e descongelamento. Sendo posteriormente, incubados por 1 hora em "banho seco" a $60^{\circ}$

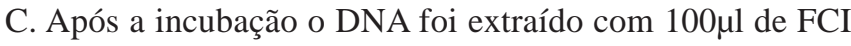
(fenol: clorofórmio: álcool isoamílico 25:24:1) e precipitado pela adição de 2 volumes de etanol absoluto (por 24 horas a $4^{\circ} \mathrm{C}$ ). Após centrifugação (12,000 x g por $5 \mathrm{~min}$ ), o precipitado foi ressuspendido com 50 $\mu \mathrm{l}$ de TE (Tris $10 \mathrm{mM}$, EDTA 0,5 mM).

Amplificação do gene 16S do DNA mitocondrial e sequenciamento. Cada amostra de DNA individualmente obtida foi utilizada para amplificação do $16 \mathrm{~S}$, com os iniciadores (primers) descritos por Lyman et al. (1999).

As reações de amplificação do $16 \mathrm{~S}$ foram conduzidas por 35 ciclos $\left(95^{\circ} \mathrm{C}\right.$ por 45 segundos, $55^{\circ} \mathrm{C}$ por 1 minuto, e $72^{\circ} \mathrm{C}$ por 1 minuto) e seus produtos (ao redor de 500 pares de base) foram visualizados após eletroforese em gel de agarose 1,5\% corado com brometo de etídeo. Os fragmentos obtidos foram então seqüenciados diretamente (sem purificação do produto da PCR), em ambas as direções com os mesmos primers usando o sistema ABI Prism dGTP BigDye Terminator Ready Reaction Kit (Perkin Elmer, Foster City, CA).

Análise das seqüências. Foram obtidas seqüências parciais do gene $16 \mathrm{~S}$ dos espécimes estudados, que foram alinhadas usando CLUSTAL W software (1.60) (Thompson et al. 1994). As sequiências estão disponibilizadas no GenBank (números de acesso na Tabela I).

As sequiências obtidas a partir das colônias do SESA foram comparadas com outras obtidas do GenBank que foram previamente cortadas e adaptadas para número de bases semelhante ao das primeiras.

Para o alinhamento, foi utilizado o programa MEGA software (Molecular Evolutionary Genetics Analysis, version 3.01) (Kumar et al. 2003) e uma matriz de divergência foi gerada. As relações entre as espécies foram avaliadas por três métodos: Neighbor Joining (NJ) com distância Kimura dois 
parâmetros, 1000 replicações no teste de "bootstrap" (MEGA); Máxima Parcimônia (MP) com 1000 réplicas de "bootstrap" e opção de busca "branch-and-bound”; e Máxima Verossimilhança (ML), utilizando o programa PHYLIP: (Phylogeny Inference Package, version 3.6) (Felsenstein 2004). As três árvores foram enraizadas com seqüências de $16 \mathrm{~S}$ de Arilus cristatus Linaeus, 1763 (Hemiptera: Reduviidae: Harpactorinae) e Oncerotrachelus sp. Stål, 1868 (Hemiptera: Reduviidae: Saicinae), como grupo externo (número de acesso no GenBank/EMBL na Tabela 2) por se tratarem de membros da Família Reduviidae, à qual os triatomíneos pertencem e terem sido utilizados por Lyman et al. (1999).

\section{RESULTADOS EDISCUSSÃO}

Foram obtidas 39 seqüências de nucleotídeos de fragmentos do gene $16 \mathrm{~S}$ do DNA mitocondrial de 14 espécies dos gêneros Triatoma, Panstrongylus e Rhodnius aos quais pertencem reconhecidos vetores da doença de Chagas na América Latina. As sequiências geradas variaram entre 311 (pares de bases) pb, para $R$. pictipes, a 317 pb em $T$. sordida (CTA 20C e 26C) (dados não mostrados).

Estes fragmentos foram alinhados com seqüências do mesmo gene para outros triatomíneos e também de $A$. cristatus e Oncerotrachelus sp. Stål, 1868. Uma matriz de distância foi construída utilizando o programa MEGA e com estes dados uma árvore de distâncias foi gerada (Fig. 1). Embora árvores por três métodos (Neighbour-Joinig, Máxima Parcimônia e, Máxima Verossimilhança) foram geradas, as espécies foram basicamente mantidas nos mesmos "clusters" ou clados em todas as árvores e, portanto, a árvore de distância foi a melhor escolha para ser mostrada e discutida neste estudo.

A árvore de distância demonstra a clara parafilia das tribos Rhodniini e Triatominini, corroborando com dados obtidos por Monteiro et al. (2000) usando CitB, D2 e LR, como marcadores, Hypša et al. (2002) que utilizaram 12S e 16S, e Paula et al. (2005) com seqüências de $16 \mathrm{~S}$ depositadas no GenBank.

Em Rhodniini, obtivemos um clado composto pelo relacionamento ( $R$. domesticus $(R$. neglectus $1 \mathrm{~A}(R$. prolixus $3 \mathrm{~A}$ (R. prolixus3B - R. prolixus 4 - R. robustus 11 (R. prolixus $29 \mathrm{~B}$ e C))))). $R$. prolixus $(3 ; 4)$ e $R$. robustus 11 não apresentaram divergência nas seqüências dos fragmentos do gene $16 \mathrm{~S}$ obtidas. Porém, R. prolixus 29 (Faculdade de Medicina) foi o mais divergente do grupo, variando de $0,3 \%$ a $0,6 \%$ e posicionando-se em ramo separado. Tal topografia corrobora a hipótese de $R$. prolixus e $R$. robustus tratarem-se de linhagens distintas de espécie única adaptadas a ecótopos diferentes (Harry et al. 1992; Harry 1993; Solano et al. 1996) mas pode sugerir também, um possível erro de identificação das colônias de $R$. prolixus $(3 ; 4)$ ou $R$. robustus, uma vez que, Monteiro et al. (2000) confirmaram a validade dos dois táxons.

Em análises mais recentes mostrou-se ainda, que o ramo acima está mais proximamente relacionado a Psammolestes tertius e P.coreodes do que ao relacionamento constituído por (R. pictipes (R. stali - R. brethesi)) (Hypša et al. 2002; Paula et al. 2005).

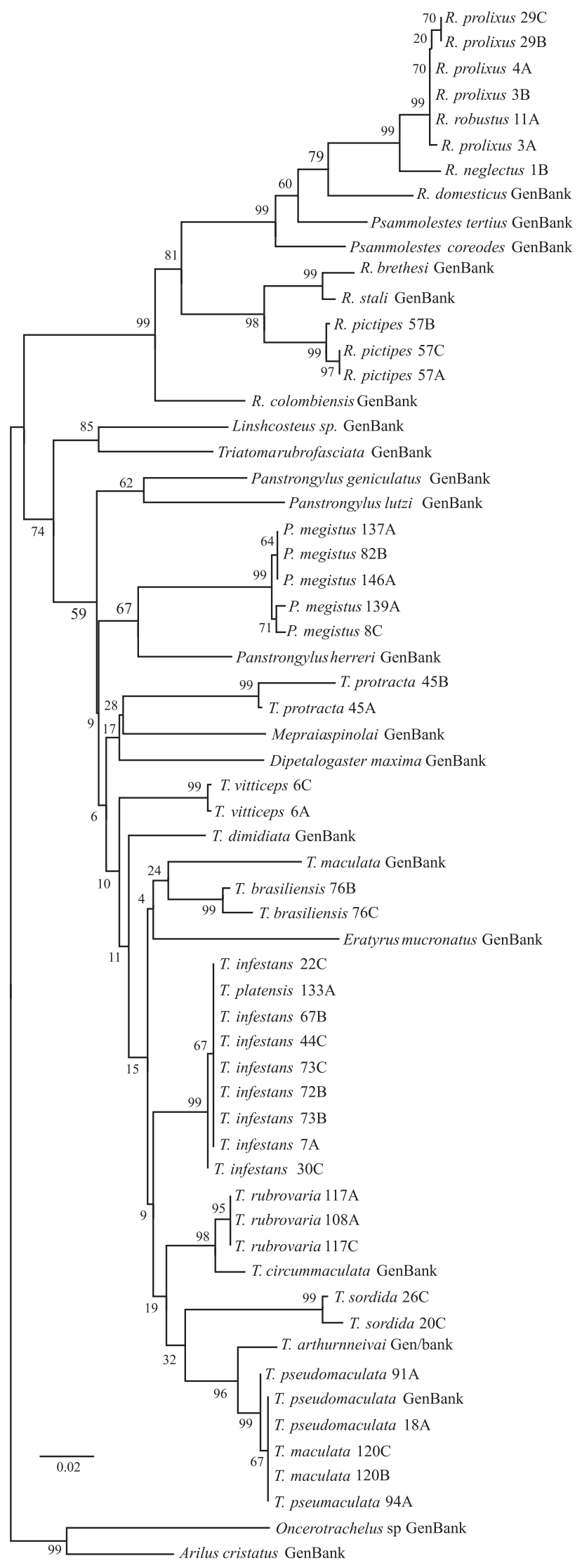

Fig. 1. Árvore de similaridade inferida das distâncias do gene 16S do DNAr mitocondrial entre triatomíneos de colônias mantidas pelo SESA - USP e GenBank. Método "Neighbor Joining", Kimura 2-parâmetros, 1000 replicações no teste "bootstrap" (programa MEGA). 
Tabela I. Lista de espécies de triatomíneos dos gêneros Rhodnius, Panstrongylus e Triatoma examinados por meio de fragmentos do gene 16S do DNAr mitocondrial, sexo e estádio, número de acesso no GenBank, procedência e código da colônia (CTA), e início da mesma, mantidas pelo SESA - USP, Araraquara-SP. A-ninfa, B-fêmea, C-macho.

\begin{tabular}{|c|c|c|c|c|c|}
\hline Espécie & Sexo Estádio & GenBank & Procedência & Colônia & Início \\
\hline R. neglectus & $\mathrm{B}$ & EU822951 & Guaíra - SP - Brasil & 001 & $14 / 03 / 1969$ \\
\hline \multirow[t]{2}{*}{$R$. prolixus } & A & EU822952 & Colômbia & 003 & $21 / 12 / 1976$ \\
\hline & $\mathrm{B}$ & EU822953 & & & \\
\hline R. prolixus* & $\mathrm{C}$ & EU822954 & Colômbia & 004 & $21 / 12 / 1976$ \\
\hline \multirow[t]{2}{*}{ R. prolixus } & $\mathrm{B}$ & EU827235 & Faculdade de Medicina Ribeirão Preto & 029 & $01 / 05 / 1967$ \\
\hline & $\mathrm{C}$ & EU827236 & & & \\
\hline$R$. robustus & A & EU827206 & Madre de Dios - Perú & 011 & $30 / 08 / 1973$ \\
\hline \multirow[t]{3}{*}{ R. pictipes } & A & EU827215 & Jacundá - PA - Brasil & 057 & 20/06/1978 \\
\hline & $\mathrm{B}$ & EU827216 & & & \\
\hline & $\mathrm{C}$ & EU827217 & & & \\
\hline P. megistus & $\mathrm{C}$ & EU827205 & Almino Alfonso - RN - Brasil & 008 & $28 / 02 / 1980$ \\
\hline P. megistus & $\mathrm{B}$ & EU827224 & Araraquara - SP - Brasil & 082 & S.I. $* *$ \\
\hline P. megistus & A & EU827233 & São Felipe - BA- Brasil & 137 & $12 / 09 / 1992$ \\
\hline P. megistus & A & EU827234 & Sta. Maria do Cambuçá - PE- Brasil & 139 & $18 / 07 / 1977$ \\
\hline P. megistus & A & EU835979 & Boca da Mata - AL- Brasil & 146 & 04/03/1980 \\
\hline \multirow[t]{2}{*}{ T. brasiliensis } & $\mathrm{B}$ & EU827222 & São João do Piauí e Conceição do Canindé- PI- Brasil & 076 & $20 / 01 / 1980$ \\
\hline & $\mathrm{C}$ & EU827223 & & & \\
\hline T. infestans & A & EU827204 & Mambaí - GO- Brasil & 007 & $28 / 02 / 1980$ \\
\hline T. infestans & $\mathrm{C}$ & EU827209 & Frutal - MG- Brasil & 022 & 22/09/1977 \\
\hline T. infestans & $\mathrm{C}$ & EU827211 & Tucuman - Argentina & 030 & $24 / 10 / 1973$ \\
\hline T. infestans & $\mathrm{C}$ & EU827212 & Cochabamba - Bolívia & 044 & 05/09/1972 \\
\hline T. infestans & $\mathrm{B}$ & EU827219 & Santa Cruz - Bolívia & 072 & $12 / 07 / 1978$ \\
\hline T. infestans & $\mathrm{B}$ & EU827218 & Tanhaçu - BA- Brasil & 067 & 07/12/1977 \\
\hline \multirow[t]{2}{*}{ T. infestans } & $\mathrm{B}$ & EU827221 & Gloria de Dourados - MS- Brasil & 073 & $12 / 09 / 1979$ \\
\hline & $\mathrm{C}$ & EU827220 & & & \\
\hline \multirow[t]{2}{*}{ T. maculata } & $\mathrm{B}$ & EU827230 & Cerro Galan - Venezuela & 120 & $19 / 12 / 1979$ \\
\hline & $\mathrm{C}$ & EU827231 & & & \\
\hline T. pseudomaculata & A & EU827207 & Pocinhos - PB- Brasil & 018 & $22 / 08 / 1977$ \\
\hline T. pseudomaculata & A & EU827225 & Ceará- Brasil & 091 & $06 / 11 / 1975$ \\
\hline T. pseudomaculata & A & EU827226 & Teofelândia - BA- Brasil & 094 & $11 / 03 / 1977$ \\
\hline T. platenses & A & EU827232 & La Pampa - Argentina & 133 & $21 / 07 / 1984$ \\
\hline \multirow[t]{2}{*}{ T. protracta } & A & EU827213 & Monte Del Diablo - California - EUA & 045 & $16 / 10 / 1972$ \\
\hline & $\mathrm{B}$ & EU827214 & & & \\
\hline T. sordida & $\mathrm{C}$ & EU827208 & Guaíra - SP- Brasil & 020 & 26/08/1977 \\
\hline T. sordida & $\mathrm{C}$ & EU827210 & Tanhaçu - BA- Brasil & 026 & $17 / 11 / 1977$ \\
\hline T. rubrovaria & A & EU827227 & Caçapava do Sul - RS- Brasil & 108 & $02 / 05 / 1983$ \\
\hline \multirow[t]{2}{*}{ T. rubrovaria } & A & EU827228 & Pelotas - RS- Brasil & 117 & $24 / 03 / 1983$ \\
\hline & $\mathrm{C}$ & EU827229 & & & \\
\hline \multirow{2}{*}{ T. vitticeps } & A & EU827202 & Guarapari - ES- Brasil & 006 & 08/11/1979 \\
\hline & $\mathrm{C}$ & EU827203 & & & \\
\hline
\end{tabular}

* Linhagem: olhos vermelhos - ** Sem informação.

R. colombiensis situou-se em ramo mais basal em relação aos outros Rhodiniini. Em filogenias anteriormente publicadas (Hypša et al. 2002; Paula et al. 2005) esta espécie aparece relacionada a $R$. pallescens enquanto, $R$. ecuadoriensis posicionou-se em ramo basal ao da Tribo. A não inclusão dessas duas espécies nesta análise justifica a posição basal de $R$. colombiensis na presente árvore.

Em Triatominini, ficou demonstrada a parafilia de Panstrongylus formando clados distintos $P$. geniculatus com $P$. lutzi basal ao ramo P. herreri com P. megistus e este, mais próximo a Dipetalogaster maxima e aos "Triatomas" Norteamericanos do que aos da América do Sul. D. maxima aparece basal em relação a o ramo $P$. herreri-P. megistus. As distâncias entre as populações de P. megistus variaram entre $0,3 \%$ e $0,6 \%$ confirmando derivação distinta para esse gênero observada também por Garcia et al. (2001), utilizando seqüências combinadas dos genes $12 \mathrm{~S}$, citocromo oxidase I (COI) e 16S), Garcia et al. (2003) usando 12S e 16S e Marcilla et al. (2002) com o uso de ITS-2, como marcadores. Esse resultado corrobora com a hipótese de origem do clado neotropical de Triatomini a partir de ancestrais das linhagens neárticas, ou ainda, por derivação direta de um dos membros já constituídos, T. rubida, por exemplo, semelhantemente ao que supostamente tenha ocorrido aos Triatominae do Velho Mundo (Patterson et al. 2001; Hypša et al. 2002; Marcilla et al. 2002; Sainz et al. 2004). 
O ramo formado por T. pseudomaculata com T. maculata apresentou divergência nula e corrobora os resultados obtidos por Sainz et al. (2004) que, usando também $16 \mathrm{~S}$, obtiveram diferença que variou de $0,2-0,8 \%$ e que sustentam nossos resultados para T. pseudomaculata 91 (Ceará) que foi $0,3 \%$ divergente dos demais e formou um ramo consistente com $T$. arthurneivai que não foi testado pelos referidos autores.

Para Sainz et al. (2004) a inclusão de T. arthurneivai na análise poderia ajudar a resolver a questão desse relacionamento. Porém, quando incluímos em nossa análise sequiências de T. maculata e T. pseudomaculata obtidas do GenBank verificamos que T. maculata do SESA (CTA-120) manteve-se associado aos $T$. pseudomaculata do SESA enquanto, o T. maculata do GenBank relacionou-se ao $T$. brasiliensis. Esse resultado coincide com Garcia et al. (2001), Hypša et al. (2002) e Paula et al. (2005) que mostraram divergências significativas entre $T$. maculata e $T$. pseudomaculata que se posicionaram em ramos independentes e muito distantes entre si.

Segundo Carbajal de La Fuente et al. (2008) as discordâncias entre os resultados exibidos por Sainz et al. (2004) e aqueles obtidos por Garcia et al. (2001), Hypša et al. (2002) e Paula et al. (2005) estão relacionados a algum erro de identificação dos espécimes utilizados por Sainz et al. (2004) como T. maculata procedente de Sergipe, que seriam de fato T. pseudomaculata. No Brasil, T. maculata ocorre apenas no Estado de Roraima (Lent \& Wygodzinsky 1979; Carcavallo et al. 1998).

Tais erros são consequiências dessas duas espécies serem de difícil separação pelos caracteres morfológicos dos adultos e as chaves dicotômicas de Lent \& Wygodzinsky (1979) e de Carcavallo et al. (1997) atribuírem grande peso a esses caracteres, principalmente os cromáticos, que são inconsistentes com a grande variedade fenotípica que esses caracteres apresentam na maioria das espécies (Carbajal de La Fuente et al. 2008).

Outro motivo também, seria a ocorrência de híbridos produzidos pelos cruzamentos entre indivíduos de colônias de T. maculata e T. pseudomaculata (Belisário et al., 2007), principalmente por que T. pseudomaculata, foi erroneamente identificada por mais de um século, antes de ser descrita por Corrêa \& Espínola (1964), a partir de insetos procedentes do Estado do Ceará, Brasil e continuaram sendo confundidas, principalmente pelas secretarias de Saúde, mesmo após os trabalhos de Lent \& Jurberg (1978), Lent \& Wygodzinsky (1979) e de Gonçalves et al. (1985) que confirmam as diferenças encontradas, na genitália externa, nos ovos e entre as ninfas das duas espécies.

Ambas são encontradas nos ambientes domésticos e peridomésticos em geral, associadas a aves. Schofield (1988) sustenta a provável evolução das mesmas a partir de populações alopátricas originadas de uma ancestral comum por dispersão passiva.

Em análises isoenzimática, citogenéticas e morfométricas da cabeça, incluindo as antenas, do tórax e do abdome ficou demonstrado que é possível distinção completa de T. maculata,
Tabela II. Espécies estudadas e correspondente número de acesso de seqüências do gene 16S do DNA mitocondrial de espécies de insetos da Família: Reduviidae, obtidas no GenBank (setembro de 2007) para comparação com as seqüências geradas nesse estudo.

\begin{tabular}{lc}
\hline Espécie & Número de acesso \\
\hline Oncerotrachelus sp & AY252739 \\
Arilus cristatus & AF045712 \\
Rhodnius domesticus & AY035440 \\
Rhodnius stali & AY035437 \\
Rhodnius brethesi & AF045710 \\
Rhodnius colombiensis & AY035438 \\
Psammolestes tertius & AY035439 \\
Psammolestes coreodes & AF045708 \\
Triatoma maculata & AY035465 \\
Triatoma pseudomaculata & AY035461 \\
Triatoma dimidiata & AF045695 \\
Triatoma circummaculata & AF021188 \\
Triatoma arthurneivai & AY035460 \\
Triatoma rubrofasciata & AY035468 \\
Panstrongylus lutzi & AY035449 \\
Panstrongylus geniculatus & AF394593 \\
Panstrongylus herreri & AY185833 \\
Dipetalogaster maximus & AF045699 \\
Eratyrus mucronatus & AY185832 \\
Mepraia spinolai & AF324518 \\
Linshcosteus sp. & AF394595 \\
\hline
\end{tabular}

T. pseudomaculata e T. arthurneivai, sendo o relacionamento evolutivo da primeira em relação às últimas questionado (Santos et al., 2007; Carbajal de La Fuente et al. 2008).

O T. maculata do SESA (CTA 120) está rotulado como procedente de Cerro Galan, Venezuela. Essa localidade está inserida na área de ocorrência natural da espécie. Desta forma, um erro de identificação é pouco provável. No entanto, devido à longevidade dessa colônia (iniciada em 1979), não podemos descartar a hipótese de hibridação, por qualquer motivo relacionado à sua manutenção no insetário, com membros de colônias de T pseudomaculata comprometendo os resultados deste trabalho.

As espécies de T. infestans (CTA 044 e 072) procedentes de Cochabamba e Santa Cruz (Bolívia) formaram ramo com as procedentes de Glória de dourados (CTA 073) e Mambaí (CTA 007), ambas do Estado de Goiás, Frutal (CTA 022) de Minas Gerais e Tanhaçú (CTA 067) de Bahia, Brasil, observando-se, também, inclusão de T. platensis (CTA 133) nesse ramo, havendo divergência nula dessa em relação aos $T$. infestans mencionados. No entanto, $T$. infestans (CTA 030) procedente de Tucuman, Argentina, divergiu 0,3\% das demais, formando ramo basal a este clado. A similaridade genética entre as colônias do Brasil e Bolívia pode ser explicada, pois, admiti-se que a região andina de Cochabamba, Bolíva seja o centro de dispersão da espécie que teria atingido o território brasileiro durante o século XX, passivamente, por meio da imigração humana (Schofield 1988). Esse argumento leva em consideração a ocorrência de populações silvestres de T. infestans naquela área. No entanto, observações recentes revelaram a existência 
de populações silvestres também na região do Chaco que abrange o sudeste da Bolívia, Paraguai e norte da Argentina onde está situada a Província de Tucuman (Noireau et al. 2005; Richer et al.2007) o que dá sustento à maior divergência desta para as colônias bolivianas e brasileiras.

A similaridade genética entre T. infestans e T. platensis já foi demonstrada anteriormente tanto, por apresentarem híbridos naturais viáveis (Perez et al. 2005) quanto por dados moleculares: Garcia et al. (2001) pelo uso de COI, $12 \mathrm{~S}$ e $16 \mathrm{~S}$ e também, por Bargues et al. (2006) usando o primeiro espaçador interno transcrito (ITS-1). Estas espécies estão mais relacionadas entre si do que com $T$. delpontei. Estas três espécies juntamente a T. melanosoma, T. rubrovaria, $T$. carcavalloi e $T$. infestans Darck Morphs, pertencem ao complexo T. infestans Carcavallo et al. (2000). No entanto, Monteiro et al. (1999) com base na análise de fragmentos de citocromo b (citb), evidenciou que tanto T. melanosoma quanto T. infestans Darck Morphs Andinos referem-se a variações fenotípicas de T. infestans.

T. circummaculata e T. rubrovaria (CTA 108; 117, A e C) formaram clado independente, mais derivado de $T$. infestans $\mathrm{e}$ T. platensis o que pode evidenciar separação mais recente. Tal topografia tem amparo nos resultados de Garcia et al. (2001). Os resultados observados para $T$. rubrovaria (108) e $T$. rubrovaria (CTA 117, A e C) com divergência nula, mostram não haver variação geográfica nas populações de Caçapava do Sul e Pelotas, pelo menos quanto ao gene testado.

Resultados inesperados com distância acima de $1 \%$, foram obtidos para os ramos de $T$. sordida $20 \mathrm{C}$ e $T$. sordida $26 \mathrm{C}$, procedentes respectivamente de Guairá-SPe Tanhaçú-BA, com 1,0\% de divergência e T. brasiliensis B e T. brasiliensis C, ambos da colônia registrada sob o CTA-076 (1,3\%) cuja procedência refere-se aos municípios de São João do Piauí e Conceição do Canindé situados no Estado do Piauí, Brasil. No entanto, estão dentro daqueles atribuídos a populações geográficas $(1,3 \% \pm 0,9 \%)$ segundo Dujardin et al. (2002).

Quanto à divergência genética dos T. brasiliensis (CTA 076, B e C), os municípios de São João do Piauí ( $8^{\circ} 18^{\prime} 45^{\prime \prime}$ ' $; 42^{\circ}$ $\left.11^{\prime} 15^{\prime \prime O}\right)$ e Conceição do Canindé (756' 15"S; 41³3'45"O) estão ambos situados no sudeste piauiense, área de predomínio geológico da Serra da Capivara e embora as altitudes estejam próximas, $222 \mathrm{~m}$ para São João do Piauí e $252 \mathrm{~m}$ para Conceição do Canindé, há muitas barreiras geográficas, determinadas pela presença de montes de cerca $600 \mathrm{~m}$ de altitude que podem sustentar a diferença observada entre os dois membros da colônia testada por ser o $16 \mathrm{~S}$ um gene mitocondrial e assim, ser transmitido, sem recombinação significativa, das mães para a prole (Abad-Franch \& Monteiro 2005). Desta forma, é provável que esses insetos descendam de fêmeas distintas pertencentes às linhagens fundadoras da colônia desta colônia.

A posição basal no clado correspondente aos Triatomas sul-americanos de T. vitticeps (Stål 1859) necessita ser melhor avaliada em análises futuras. Esse triatomíneo, ocorrente nos estados brasileiros de Minas Gerais, Bahia, Espírito Santo e Rio de Janeiro é freqüentemente coletado pelos moradores de áreas rurais do Espirito Santo e incriminado pela transmissão do T. cruzi no Estado do Rio de Janeiro. Essa espécie apresentou elevada taxa de infecção natural por tripanosomatídeos semelhantes ao T. cruzi e índices elevados de domiciliação para o Estado do Espirito Santo. (Santos et al. 2006).

Esta análise evidencia a importância do $16 \mathrm{~S}$ como marcador de espécies de triatomíneos e sua importância em questões de sistemática e taxonomia. No entanto, os resultados aqui obtidos podem ter sofrido influências relacionadas à homogeneidade genética de cada colônia devido ao estado panmítico das mesmas.

Apesar da sua grande longevidade, admitimos que as colônias do SESA consistam um importante acervo para pesquisa. Pois, permitem fazer análises comparativas entre populações recentes com populações que não são mais encontradas como, por exemplo, os T. infestans intradomiciliares de várias localidades do Brasil que hoje se encontram erradicados.

A partir dos trabalhos de Garcia \& Powell (1998) e Stothart et al. (1998) que segundo Galvão (2003), inauguraram a era cladística molecular nos estudos sistemáticos de Triatominae resolveram-se muitos impasses relacionados ao status de algumas espécies ou relativos à espécies crípticas, no entanto, acirrou-se a discussão sobre a origem evolutiva deste grupo e as evidências para polifilia, ou parafilia ganharam mais suporte (Monteiro et al. 2004; Paula et al. 2005). Novas análises com uso de um maior número de marcadores genéticos associadas ainda, aos padrões sistemáticos clássicos de morfologia, ecologia e comportamento, devem ser adicionadas para uma decisão filogenética adequada. Uma vez que isto não tem apenas relevância sistemática, mas, sérias implicações quanto às medidas de controle das espécies de vetores (Scheafer 2003, 2005).

Agradecimentos. À FAPESP (MTM - 05/50225-2) pelo financiamento.

\section{REFERÊNCIAS}

Abad-Franch, F. \& F. A. Monteiro. 2005. Molecular research and the control of Chagas disease vectors. Anais da Academia Brasileira de Ciências 77: 437-454.

Barata, J. M. S. 1998. Macroscopic and exochorial structures of Triatominae eggs (Hemiptera, Reduviidae). Estruturas macroscópicas e exocoriais de ovos de Triatominae (Hemiptera, Reduviidae). In: R. U. Carcavallo, I. Galíndez-Girón, J. Jurberg, H. Lent - orgs.). Atlas of Chagas'disease vectors in the Americas. Atlas dos vetores da doença de Chagas nas Américas. Editora FIOCRUZ, Rio de Janeiro. Vol. II, pp. 409-448.

Bargues, M. D.; D. R. Klisiowicz; F. Panzera; F.; Noireau; A. Marcilla; R. Perez; M. G. Rojas; J. E. R. O’ConnorGonzales-Candelas; C. Galvão; J. Jurberg; R. U. Carcavallo; J. P. Durjardin \& S. MasComa. 2006. Origin and phylogeography of the Chagas disease main vector Triatoma infestans based on nuclear rDNA sequences and genome size. Infection, Genetics and Evolution 6: 42-62.

Belisário C. J.; G. C. D’Ávila Pessoa \& L. Diotaiuti. 2007. Biological aspects of crosses between Triatoma maculata (Erichson, 1848) and Triatoma pseudomaculata Corrêa \& Espínola, 1964 (Hemiptera: Reduviidae). Memórias do Instituto Oswaldo Cruz 102: 517-521. 
Carbajal de la Fuente, A. L.; F. Noireau \& S. S. Catalã. 2008. Inferences about antennal phenotype: the "Triatoma maculata complex" (Hemiptera: Triatominae) is valid? Acta Tropica 106: 16-21.

Carcavallo, R. U.; I. Galíndez Giríon; J. Jurberg; C. Galvão \& H. Lent. 1997. Pictorial keys for tribes, genera and species of the subfamily Triatominae. In: Carcavallo, R. U., I. Galíndez Giríon, J. Jurberg \& H. Lent. (Eds.), Atlas of Chagas Disease Vectors in the Americas, vol. 3. FIOCRUZ, Rio de Janeiro, Brazil, pp. 107-244.

Carcavallo, R. U.; S. I. Curto de Casas; I. A. Sherlock ; I. Galílndez Giríon; J. Jurberg; C. Galvão; C. A. Mena-Segura \& F. Noireau. 1998. Geographical distribution and alti-latitudinal dispersion. In: Carcavallo, R.U., I. Galíndez Giríon, J. Jurberg \& H. Lent. (Eds.), Atlas of Chagas Disease Vectors in the Americas, vol. 3. FIOCRUZ, Rio de Janeiro, Brazil, pp. 747-793.

Carcavallo, R. U.; J. Jurberg; H. Lent; F. Noireau \& C. Galvão. 2000. Phylogeny of the Triatomionae (Hemiptera: Reduviidae). Proposals for taxonomic arrangements. Entomologia y Vectores 7: 1-99.

Carcavallo, R. U.; J. Jurberg; H. Lent; C. Galvão; M. Steindel \& C. J. C. Pinto. 2001. Nova espécie do complexo oliveriai (nova denominação para o complexo matogrossensis) (Hemiptera, Reduviidae, Triatominae) do Estado do Rio Grande do Sul, Brasil. Memórias do Instituto Oswaldo Cruz 96: 77-79.

Corrêa R. R. \& H. N. Espínola. 1964. Descrição de Triatoma pseudomaculata, nova espécie de Triatomíneo de Sobral, Ceará. Arquivos de Higiene e Saúde Pública 29: 115-127.

Costa, J.; A. M. Argolo \& M. Felix. 2006. Redescription of Triatoma melanica Neiva \& Lent, 1941, new status (Hemiptera: Reduviidae: Triatominae) Zootaxa 1385: 47-52.

Costa, J. \& M. Felix. 2007. Triatoma juazeirensis sp. nov. from the state of Bahia, Northeastern Brazil (Hemiptera: Reduviidae: Triatominae). Memórias do Instituto Oswaldo Cruz 102: 8790.

Felsenstein, J. 2004. Evolutionary trees from DNA sequences: a maximum likelihood approach. Journal of Molecular Evolution 17: $368-376$.

Forero, D.; C. Weirauch \& M. Baena. 2004. Synonymy of the reduviid (Hemiptera: Heteroptera) genus Torrealbaia (Triatominae) with Amphibolus (Harpactorinae), with notes on Amphibolus venator. (Klug, 1830). Zootaxa 670: 1-12.

Galvão, C.; R. Carcavallo; D. Silva Rocha \& J. Juberg. 2003. A cheklist of the current species of the subfamily Triatominae Jeannel, 1919 (Hemiptera, Reduviidae) and their geographical distribution, with nomenclatural and taxonomic notes. Zooata 202: 1-36.

Galvão, C. 2003. A sistemática de Triatomíneos (Hemikptera, Reduviidae), de De Geer ao DNA. In: II Encuentro Nacional de Entomología Médica y Vetrinária. VII Jornadas de Zoologia del Uruguay, 13-17 de octubre de 2003. Montevideo Uruguay. Entomología y Vectores 10: 511-530.

Galvão, C. \& V. M. Angulo. 2006. Belminus corredori, a new species of Bolboderini (Hemiptera: Reduviidae: Triatominae) from Santander, Colombia Zootaxa 1241: 61-68.

Garcia, B. A. \& J. R. Powell. 1998. Phylogeny of species of Triatoma (Hemiptera: Reduviidae) based on mitochondrial DNA sequences. Journal of Medical Entomology 35: 232-238.

Garcia, B. A.; E. N. Moriyama \& J. R. Powell. 2001. Mitochondrial DNA sequences of Triatominae (Hemiptera: Reduviidae): Phylogenetic relationships. Journal of Medical Entomology 38: $675-683$.

Garcia, B. A.; C. Manfredi; L. Fichera \& E. L. Segura. 2003. Short report: Variation in mitochondrial $12 \mathrm{~S}$ and 16S Ribosomal DNA sequences in natural populations of Triatoma infestans (Hemiptera: Reduviidae). American Journal of Tropical Medicine and Hygiene 68: 692-694.

Gonçalves, T. C. M.; J. Jurberg; J. M. Costa \& W. de. Souza. 1985. Estudo morfológico comparativo de ovos e ninfas de Triatoma maculata (Erichson, 1848) e Triatoma pseudomaculata Corrêa \& Espínola, 1964 (Hemiptera, Reduviinae, Triatominae). Memórias do Instituto Oswaldo Cruz 80: 263-276.

Harry, M.; I. Galindez \& M. L. Cariou. 1992. Isozyme variability and differentiation between Rhodnius prolixus, $R$. robustus and $R$. pictipes, vectors of Chagas disease in Venezuela. Medical Veterinary Entomology 6: 37-43.

Harry, M. 1993. Isozymic data question the specific status of bloodsucking bugs of genus Rhodnius, vectors of Chagas disease. Transactions of the Royal Society of Tropical Medicine and Hygiene 87: 492.

Hypša, V.; D. F. Tietz; J. Zrzavy; R. O. M. Rego; C. Galvão \& J. Jurberg. 2002. Phylogeny and biogeography of Triatominae (Hemiptera: Reduviidae): molecular evidence of a New World origin of the Asiatic clade. Molecular Phylogenetics and Evolution 23: 447457.

Jurberg, J. 2003. Ferramentas usadas em taxonomia de triatomíneos: O uso múltiplo.In: II Encuentro Nacional de Entomología Médica y Vetrinária. VII Jornadas de Zoologia del Uruguay, 13-17 de octubre de 2003, Montevideo Uruguay. Entomología y Vectores 10: 497-509.

Kumar, S.; K. Tamura \& M. Nei. 2004. MEGA3: Integrated Software for Molecular Evolutionary Genetics Analysis and Sequence Alignment. Briefings in Bioinformatics 5: 150-163.

Lent, H. \& P. Wygodzinsky. 1979. Revision of the Triatominae (Hemiptera, Reduviidae), and their significance as vectors of Chagas' disease. Bulletim of America Museum of Natural History 163: $123-520$.

Lent, H. \& J. Jurberg. 1978. Estudo comparativo da genitália externa masculina de seis espécies de Triatoma Laporte, 1832 que mais freqüentemente habitam o domicílio humano no Brasil (Hemiptera, Reduviidae). Revista Brasileira de Biologia 38: 931-944.

Lyman, D. F.; F. A. Monteiro; A. A. Escalante; C. Cordon-Rosales; D. N. Wesson; J. P. Dujardin \& C. B. Beard. 1999. Mitochondrial DNA sequence variation among Triatominae vectors of Chagas' disease. American Journal of Tropical Medicine and Hygiene 60: $377-386$.

Marcilla, A.; M. D. Bargues; F. Abad-Franch; F. Panzera; R. U. Carcavallo; F. Noireau; C. Galvão; J. Jurberg; M. A. Miles; J. P. Durjardin \& S. Mas-Coma. 2002. Nuclear rDNA ITS-2 sequences reveal polyphyly of Panstrongylus species (Hemiptera: Reduviidae: Triatominae), vectors of Trypanosoma cruzi. Infection, Genetics and Evolution 1: 225-235.

Martínez, E.; T. Chávez; D. Sossa; R. Aranda; V. Benigno \& P. Vidaurre. 2007. Triatoma boliviana sp. $\mathrm{n}$. de los valles subandinos de La Paz, Bolívia (Hemiptera: Reduviidae: Triatominae), similar a Triatoma nigromaculata Stål, 1859. Boletín del Instituto de Investigación en Salud y Desarrollo 3: 1-10.

Monteiro, F. A.; R. Pérez; F. Panzera; J. P. Dujardin; C. Galvão; D. Rocha; F. Noireau; C. Schofield \& C. B. Beard. 1999.Mitochondrial DNA Variation of Triatoma infestans Populations and its Implication on the Specific Status of T. melanosoma. Memórias do Instituto Oswaldo Cruz 94: 229-238.

Monteiro, F. A.; D. M. Wesson; E. M.; Dotson; C. J. Schofield \& C. B. Beard. 2000. Phylogeny and molecular taxonomy of the Rhodniini derived from mitochondrial and nuclear DNA sequences. American Journal of Tropical Medicine and Hygiene 62: 460-465.

Monteiro, F. A.; A. A. Escalante \& C. B. Beard. 2001. Molecular tools and triatomine systematics: a public health perspective. Trends in Parasitology 17: 344-347.

Monteiro, F. A.; M. J. Donnelly; C. B. Beard \& J. Costa. 2004. Nested clade and phylogeographic analyses of the Chagas disease vector Triatoma brasiliensis in Northeast Brazil. Molecular Phylogenetics Evolution 32: 46-56.

Noireau, F.; M. G. R. Cortez; F. A. Monteiro; A. M. Jansen \& F. Torrico. 2005. Can wild Triatoma infestans foci in Bolivia jeopardize Chagas disease control efforts? Trends in Parasitology 21: 7-10.

Patterson, J. S.; C. J. Schofield; J. P. Durjardin; M. A. Miles. 2001. Population morphometric analysis of the tropicopolitan bug Triatoma rubrofasciata and relationships with old world species of Triatoma: evidence of new world ancestry. Medicine Veterinay and Entomology 15: 443-451.

Paula, A. S. de; L. Diotaiuti \& C. J. Schofield. 2005, Testing the sistergroup relationship of the Rhodniini and Triatomini (Insecta: 
Hemiptera: Reduviidae: Triatomiae). Molecular Phylogenetics Evolution 35: 712-718.

Poinar, J. R. G. 2005. Triatoma dominicana sp. n. (Hemiptera: Reduviidae:Triatominae), and Trypanosoma antiquus sp. $\mathrm{n}$. (Stercoraria: Trypanosomatidae), the First Fossil Evidence of a Triatomine-Trypanosomatid Vector Association. Vector-borne and Zoonotic Diseases 5: 72-81.

Richer W.; P. Kengne; M. R. Cortez; M. M. Perrineau; A. Cohuet; D. Fontenille \& F. Noireau. 2007. Active dispersal by wild Triatoma infestans in Bolivian Andes. Tropical Medicine and International Health 12: 759-764.

Sainz, A. C.; L. V. Mauro; E. N. Moriyama \& B. A. García. 2004. Phylogeny of triatomine vectors of Trypanosoma cruzi suggested by mitochondrial DNA sequences. Genetica 121: 229-240.

Sandoval, C. M.; E. Pabon; J. Jurberg \& C. Galvão. 2007. Belminus ferroae $\mathrm{n}$. sp. from the Colombian north-east, with a key to the species of the genus (Hemiptera: Reduviidae: Triatominae). Zootaxa 1443: 55-64.

Santos, C. B. dos; G. R. Leite; G. E. M. Ferreira \& A. L. Ferreira. 2006. Infecção natural de Triatoma vitticeps (Stal, 1859) por fragelados morfologicamante semelhantes ao Trypanosoma cruzi (Chagas, 1909) no Estado do Espirito Santo. Revista Brasileira de Medicina Tropical 39: 89-91.

Santos, S. M. dos; C. M. Lopes; J. P. Dujardin; F. Panzera; R. Pérez; A. L Carbajal de la Fuente; R. S. Pacheco \& F. Noireau. 2007. Evolutionary relationships based on genetic and phenetic characters between Triatoma maculata, Triatoma pseudomaculata and morphologically related species (Reduviidae: Triatominae). Infection, Genetics and Evolution 7: 469-475.

Scheafer, C. W. 2003. Triatominae (Hemiptera: Reduviidae): Systematic Questions and Some Others. Neotropical Entomology 32: 001010 (FORUM).

Scheafer, C. W. 2005. Why are the subfamly relationships of Triatomionae (Hemiptera: Reduviidae) important? Entomologia y Vectores 12: 19-35.

Schmuñis, G. A. 1999. Iniciativa del Cono Sur. In Proceedings of the Second International Workshop on Population Biology and Control of Triatominae (Schofield, C. J. and Ponce, C., eds), pp. 26-31.

Schofield, C. J. 1988. Biosystematics of Triatomiane. In: Biosystematics of Haematophagous insects (ed. M.W. Service) Systematics Association Special Volume 37, pp. 284-312. Clarendon Press, Oxford.

Schofield, C. J.; J. Jannin \& R. Salvatella. 2006. The future of Chagas disease control. Trends in Parasitology 22: 583-588.

Solano, P.; J. P. Dujardin; C. J. Schofield; C. Romana \& M. Tibayrenc. 1996. Isoenzymes as a tool for identification of Rhodnius species. Research and Reviews in Parasitology 56: 41-47.

Stothart, J. R.; Y. Yamamoto; A. Cherchi; A. L. Garcia; S. A. S. Valente; C. J. Schofield; \& M. A. Miles. 1998. A preliminary survey of mitochondrial sequence variation in Triatominae (Hemiptera: Reduviidea) using polymerase chain reaction-based single stand conformational polymorphism (SSPC) analysis and direct sequencing. Bulletin of Entomological 88: 553-560.

Thompson, J. D.; D. G. Higgins \& T. J. Gibson. 1994. CLUSTAL-W improving the sensitivity and progressive multiple sequence alignment through sequenced weighting, positions-specific gap penalties and weight matrix choice. Nucleic Acid Research 22 4673-4680.

W.H.O. 1990. Tropical Diseases 1990. TDR/CTD/HH20.1, World Health Organization.

W.H.O. 1991. Control of Chagas Disease. WHO Technical Report Series 811, World Health Organization.

W.H.O. 2002. Control of Chagas Disease. Second Report of the WHO Expert Committee. WHO Technical Report Series 905: 109.

Zahler, A. M. \& M. B. Roth. 1995. Distinct functions of SR proteins in recruitment of Ul small nuclear ribonucleoprotein to alternative 5 splice sites. Proceeding of Natural Academy of Scince. USA. 92: 2642-2646.

Recebido em 09/11/2007; aceito em 24/06/2008 